Old Dominion University ODU Digital Commons

Electrical \& Computer Engineering Faculty

Publications

Electrical \& Computer Engineering

2006

\title{
Electron Bernstein Wave Simulations and Comparison to Preliminary NSTX Emission Data
}

Josef Preinhaelter

Jakub Urban

Pavol Pavlo

Gary Taylor

Steffi Diem

See next page for additional authors

Follow this and additional works at: https://digitalcommons.odu.edu/ece_fac_pubs

Part of the Electrical and Computer Engineering Commons, and the Plasma and Beam Physics Commons

\section{Repository Citation}

Preinhaelter, Josef; Urban, Jakub; Pavlo, Pavol; Taylor, Gary; Diem, Steffi; Vahala, Linda L.; and Vahala, George, "Electron Bernstein Wave Simulations and Comparison to Preliminary NSTX Emission Data" (2006). Electrical \& Computer Engineering Faculty Publications. 27.

https://digitalcommons.odu.edu/ece_fac_pubs/27

\section{Original Publication Citation}

Preinhaelter, J., Urban, J., Pavlo, P., Taylor, G., Diem, S., Vahala, L., \& Vahala, G. (2006). Electron Bernstein wave simulations and comparison to preliminary NSTX emission data. Review of Scientific Instruments, 77(10F524), 1-4. doi: 10.1063/1.2336467 
Authors

Josef Preinhaelter, Jakub Urban, Pavol Pavlo, Gary Taylor, Steffi Diem, Linda L. Vahala, and George Vahala 


\title{
Electron Bernstein wave simulations and comparison to preliminary NSTX emission data
}

\author{
Josef Preinhaelter, Jakub Urban, and Pavol Pavlo \\ EURATOM/IPP.CR Association, Institute of Plasma Physics, 18200 Prague 8, Czech Republic \\ Gary Taylor and Steffi Diem \\ Princeton Plasma Physics Laboratory, Princeton, New Jersey 08543 \\ Linda Vahala \\ Old Dominion University, Norfolk, Virginia 23529 \\ George Vahala \\ College of William \& Mary, Williamsburg, Virginia 23185
}

(Received 7 May 2006; presented on 11 May 2006; accepted 11 July 2006; published online 12 October 2006)

\begin{abstract}
Simulations indicate that during flattop current discharges the optimal angles for the aiming of the National Spherical Torus Experiment (NSTX) antennae are quite rugged and basically independent of time. The time development of electron Bernstein wave emission (EBWE) at particular frequencies as well as the frequency spectrum of EBWE as would be seen by the recently installed NSTX antennae are computed. The simulation of EBWE at low frequencies (e.g., $16 \mathrm{GHz}$ ) agrees well with the recent preliminary EBWE measurements on NSTX. At high frequencies, the sensitivity of EBWE to magnetic field variations is understood by considering the Doppler broadened electron cyclotron harmonics and the cutoffs and resonances in the plasma. Significant EBWE variations are seen if the magnetic field is increased by as little as $2 \%$ at the plasma edge. The simulations for the low frequency antenna are compared to preliminary experimental data published separately by Diem et al. [Rev. Sci. Instrum. 77 (2006)] (c) 2006 American Institute of Physics. [DOI: 10.1063/1.2336467]
\end{abstract}

\section{INTRODUCTION}

The low magnetic fields and high plasma densities in spherical tokamaks lead to overdense plasma from which one cannot observe the standard $O$ and $X$ mode radiations emitted from the first five electron cyclotron harmonics. Hence any measured electron cyclotron emission (ECE) cannot be simply interpreted. On the other hand, electrostatic electron Bernstein waves (EBWs) are not subject to a density limit and are strongly emitted/absorbed near the electron cyclotron harmonics. On mode converting to electromagnetic waves near the upper hybrid resonance, the emitted cyclotron radiation can be attributed to electron Bernstein wave emission (EBWE) and hence to a local temperature near the cyclotron harmonic. On NSTX, there are two new steerable antennas ${ }^{1}$ which can scan the frequency ranges of $8-18$ and $18-40 \mathrm{GHz}$, respectively, as well as in directional angles. The significance of determining the frequency dependence of EBWE is that it provides important information on optimal parameters for proposed EBW heating and current drive experiments.

For our EBW modeling of the NSTX plasma, the time evolution of the magnetic field is reconstructed from EFIT, ${ }^{2}$ assuming toroidal symmetry. The evolutions of the temperature and density profiles are taken from Thomson scattering data. The position and waist of the radiation into the antennae are determined from a Gaussian beam formulation. The beam is then modeled by a symmetric distribution of rays.
The intersection of the antenna beam with the last closed flux surface determines the position of the spot. To determine the mode conversion efficiency for EBW- $X-O$ near the upper hybrid resonance, a full wave solution for a cold plane stratified plasma is obtained using adaptive finite element methods. ${ }^{3}$ From the mode conversion region, a threedimensional (3D) ray tracing code describes the propagation of EBW towards the plasma center. ${ }^{4}$ (Reciprocity between emission and absorption is assumed and we use this interchangeability often.) The radiative temperature for RayleighJeans blackbody radiation is determined by simultaneously solving the ray equations with the radiative transfer equation for each ray, ${ }^{5}$ taking into account reabsorption because of nonlocal wave damping. It should be noted that for the high frequencies under consideration here, the direct EBW- $X$ mode conversion is extremely small and plays no significant role in EBWE.

\section{DETERMINATION OF OPTIMAL ANTENNA ORIENTATION}

We wish to determine the optimal antenna orientation for the detection of ECE in typical NSTX $L$-mode plasmas. Because of the many free parameters, a computer cluster with many processing units is utilized. Using PYTHON, ${ }^{8}$ at each Thomson scattering time and for given antenna orientation and frequency, the data are distributed so that each processor 

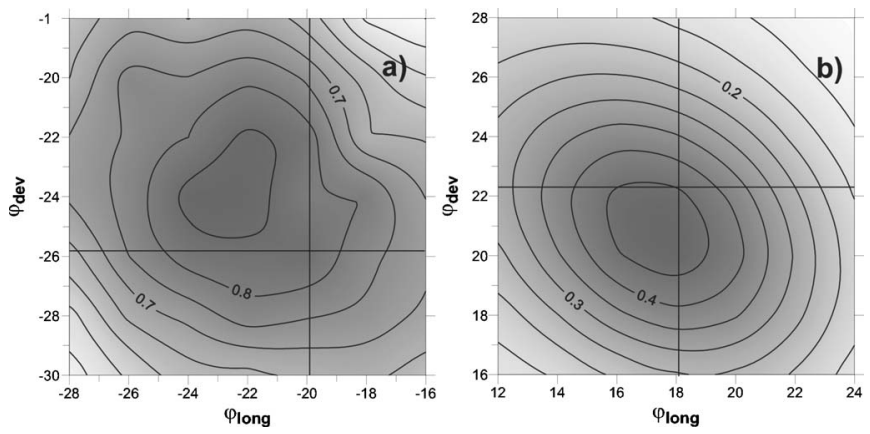

FIG. 1. The angular dependence of the EBWE (temperature in $\mathrm{keV}$ ) for NSTX shot 120278. (a) Low frequency antenna at $16 \mathrm{GHz}$ and (b) high frequency antenna at $29 \mathrm{GHz}$.

integrates the 41 rays, which model the Gaussian beam. The intensity of ECE detected by the antenna

$$
I_{\mathrm{ECE}}=\text { const } \int d S W_{\mathrm{Gauss}} C_{\mathrm{EBW}-X-O} \omega^{2} T_{\text {rad }} V_{\text {relat }},
$$

where $w_{0}$ is the beam waist radius, $W_{\text {Gauss }}=\exp \left(-2 r^{2} / w_{0}^{2}\right)$ is the Gaussian weight factor ( $r$ is the local polar coordinate for the rays in the axisymmetric Gaussian beam), $C_{\mathrm{EBW}-\mathrm{X}-\mathrm{O}}$ is the mode conversion efficiency (for high frequencies, the direct EBW- $X$ conversion is negligible), and $\omega^{2} T_{\text {rad }}$ is the RayleighJeans blackbody radiation (the radiation temperature $T_{\text {rad }}$ is defined in Ref. 5). $V_{\text {relat }}=w^{2} / w_{0}^{2}$ is the relative visible area ( $w$ is the Gaussian beam radius at the plasma surface).

In Fig. 1, we plot the angular dependence of the EBWE intensity for low [16 GHz, Fig. 1(a)] and high frequencies [ $29 \mathrm{GHz}$, Fig. 1(b)] for NSTX shot (120278) at a time where there is a flattop current in the $L$ mode. The negative angles in the low frequency shot [Fig. 1(a)] correspond to that antenna being above the equatorial plane, while the positive angles correspond to the antenna below the equatorial plane. The optimal poloidal and toroidal angles $\left(\varphi_{\mathrm{dev}}, \varphi_{\text {long }}\right)$ for the low frequency antenna are $\left(-24^{\circ},-22^{\circ}\right)$ while those for the high frequency antenna are $\left(+21^{\circ},+17^{\circ}\right)$. For the shot 120278 , these correspond well with the $N_{\|}$at the $O-X$ mode conversion at the plasma cutoff: $N_{\|}=0.6$ for $16 \mathrm{GHz}$ and $N_{\|}=0.5$ for $29 \mathrm{GHz}$ and the pitch angle to the magnetic field of about $45^{\circ}, N_{\|}=c k_{\|} / \omega$, and $\|$ is parallel to the local magnetic field. The actual experimental antenna orientation for shot 120278, with observed high EBWE, can be seen to be quite close to the optimal simulation angles (the intersection of the axis crosshairs in Fig. 1).

\section{TIME AND FREQUENCY DEVELOPMENT OF EBWE SIGNALS}

To understand the emission processes occurring within NSTX, we simulate the time and frequency evolution of the EBWE signals. In Fig. 2, we plot the time development of the radiation temperature from our simulations as well as that arising from the Thomson scattering Te data at the magnetic axis. The dips in the simulations correspond to the decrease in the conversion efficiency $C_{\mathrm{EBW}-X-O}$ due to random fluctuations in the density gradients at the plasma cutoff. These fluctuations decrease the density gradients. The emission is from the first harmonic of the electron cyclotron frequency

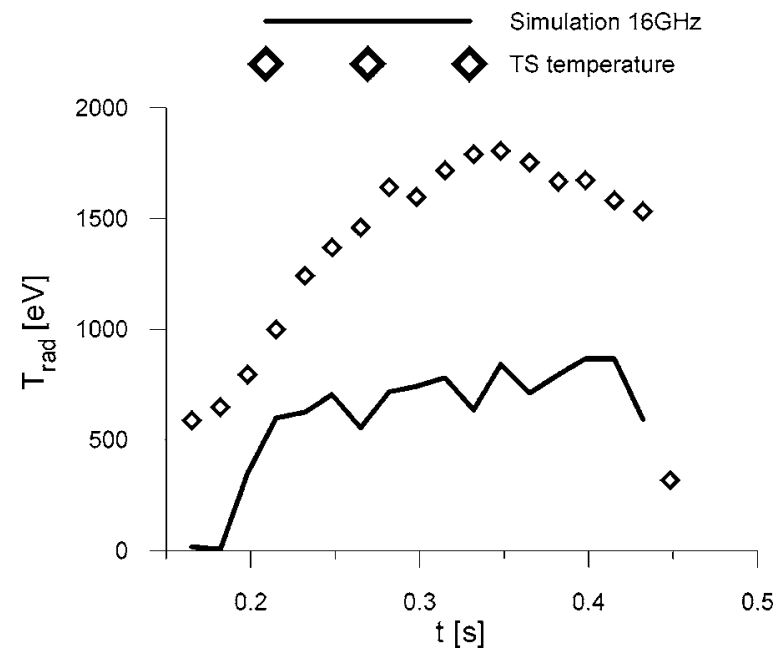

FIG. 2. The time development of EBWE at $16 \mathrm{GHz}$ for shot 120278 . TS are the Thomson scattering data. Experiment antenna orientation is $\varphi_{\mathrm{dev}}=-25.75^{\circ}, \varphi_{\text {long }}=-19.8^{\circ}$.

$\left(\omega_{\mathrm{ce}}\right)$ as can be seen from the characteristic NSTX resonances and cutoffs, shown in Fig. 3. This is further substantiated in Fig. 4, where various parameters are plotted from the upper hybrid resonance region $(R=1.45 \mathrm{~m})$ to the location of the resonance with the broadened first electron cyclotron harmonic and subsequent strong ray absorption. The intensity of EBWE is determined by the temperature in the absorption region which occurs for the first harmonic when $\left(\omega-\omega_{\mathrm{ce}}\right) / k_{\|} v_{T} \leqslant 3\left(v_{T}\right.$ is the electron thermal velocity). If one compares the simulated EBWE to the preliminary data for the measured EBWE at $16 \mathrm{GHz}$ presented in Ref. 1 we see rather good agreement. The slightly higher temperature found in our simulations probably corresponds to the fact that we have ignored the effects of the vacuum window transmission loss-which typically accounts for up to a $20 \%$

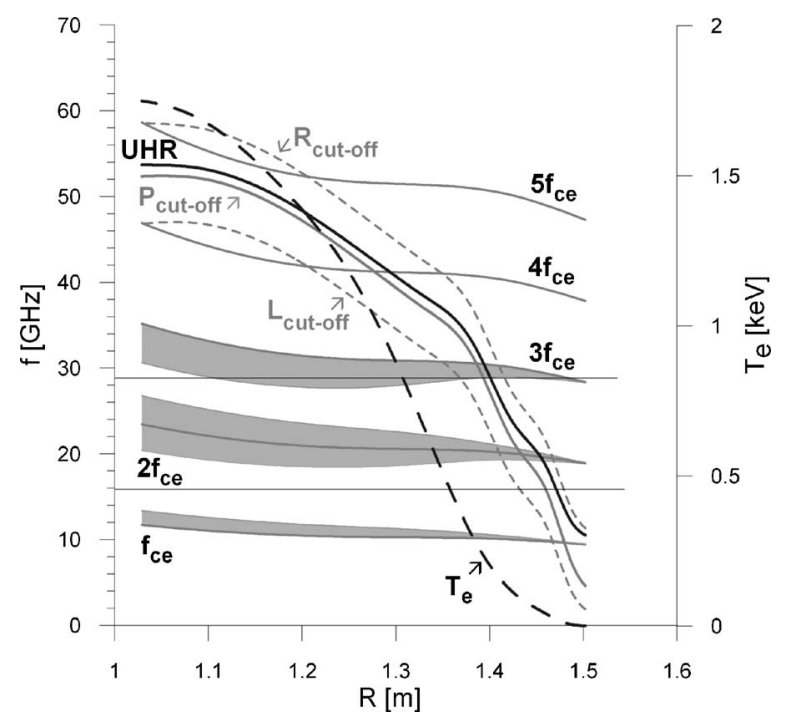

FIG. 3. The characteristic resonances and cutoffs for shot 12078. The effect of the Doppler broadening factor $1 /\left(1 \pm 3 N_{\|} v_{T} / c\right)$ on the low harmonics is depicted by the shaded regions. EBW is emitted from the boundaries of the gray strips and not from the harmonics themselves. $f_{\text {ce }}$ is the electron cyclotron frequency, $L_{\text {cutoff }}$ and $R_{\text {cutoff }}$ are the left and right cutoffs, and $P_{\text {cutoff }}$ is the plasma cutoff (Ref. 7). $R$ is the radial coordinate from the major axis. 


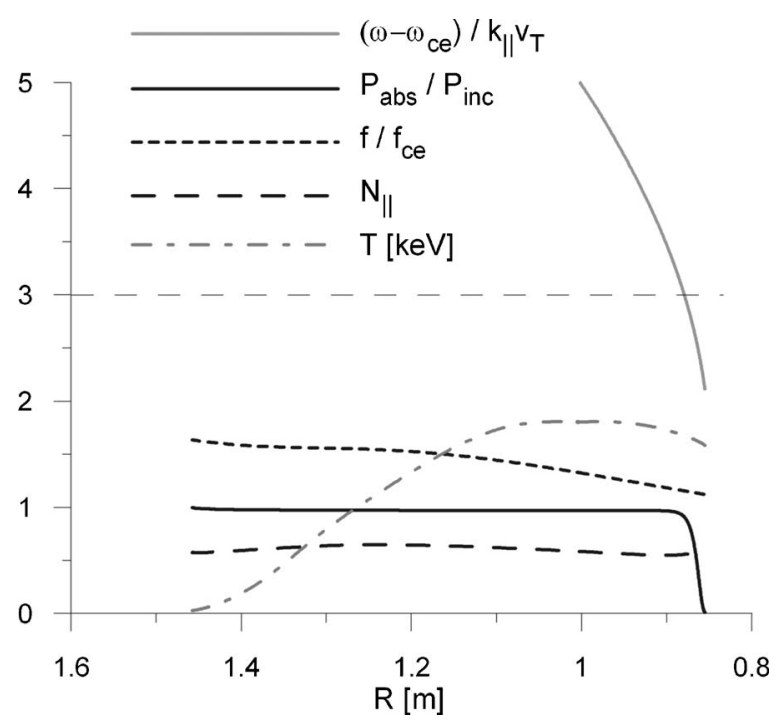

FIG. 4. The radial dependence of various parameters along the central ray as it propagates from the plasma edge $(R=1.45 \mathrm{~m})$ for shot 12078 at $16 \mathrm{GHz}$; angles are given in Fig. 2 and $t=0.348 \mathrm{~s}$. Power absorption/emission is very localized near $R=0.86 \mathrm{~m}$ where $\left(\omega-\omega_{\mathrm{ce}}\right) / k_{\|} v_{T} \leqslant 3$ and where the temperature is high.

reduction in the emission power. A more careful comparison would also require the use of a time averaged signal because in our simulations we do not include the effects of plasma turbulence.

The simulation EBWE frequency spectrum at the time of current flattop in the low frequency regime, $12 \mathrm{GHz} \leqslant f$ $\leqslant 18 \mathrm{GHz}$, is shown in Fig. 5. For frequencies $f<16 \mathrm{GHz}$, these simulations show only a slight decrease in the temperature in distinction from preliminary experimental results. ${ }^{1}$ However, there is a stronger decrease in temperature if we now include collisions (e.g., in helium $L$-mode discharges one finds a rather high $Z_{\text {eff }}$-in Fig. 5 we consider $Z_{\text {eff }}=4$ ). This correction is effective but not sufficient to match the experimental results. It should be noted that this damping could be even more prominent if one considers the radial error bars in the Thomson temperature profile and the shift in

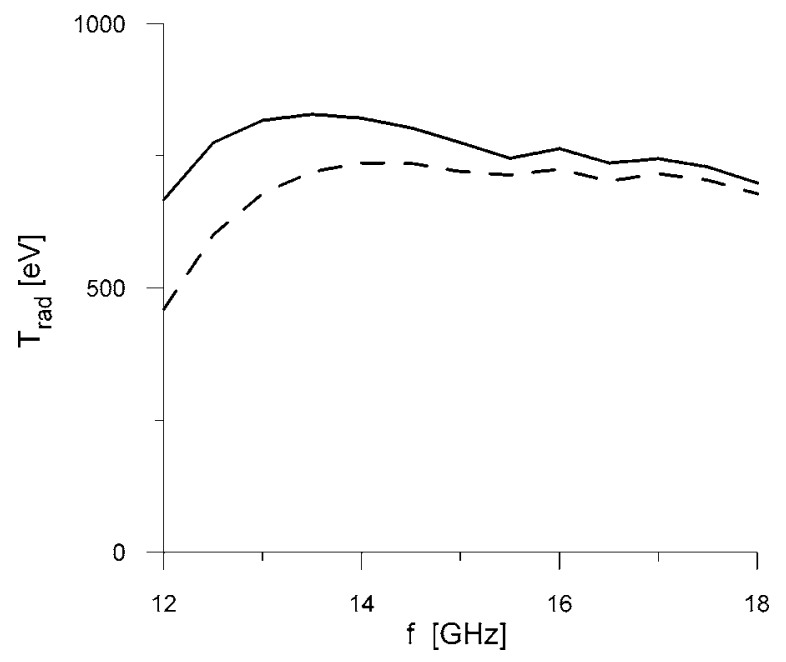

FIG. 5. The simulation frequency spectrum of EBWE in the low frequency NSTX antenna. The time and angles are given in Fig. 2. Full line: collisionless plasma; dashed line: collisional plasma with $Z_{\text {eff }}=4$.

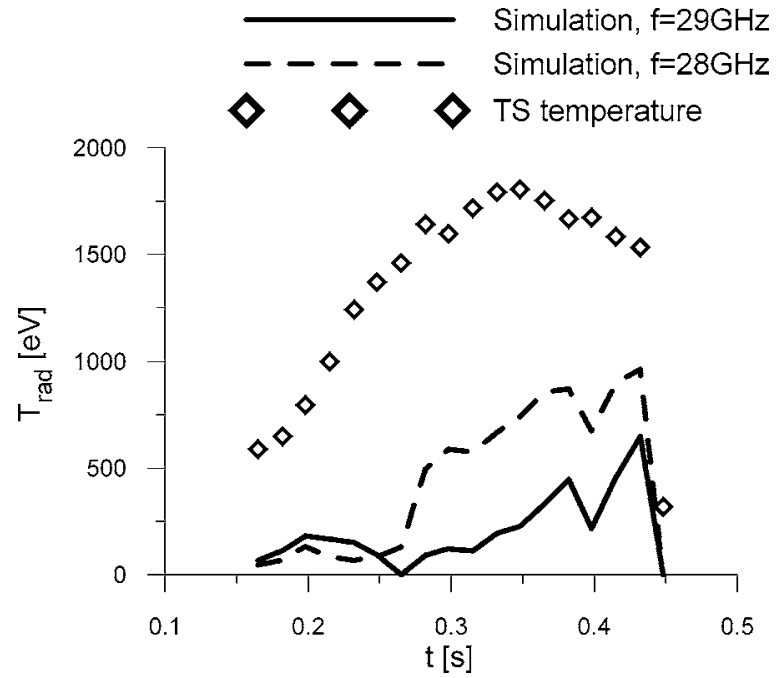

FIG. 6. A time development of EBWE at both 28 and $29 \mathrm{GHz}$ for shot 120278. TS represent Thomson scattering data. Experiment antenna orientation is $\varphi_{\mathrm{dev}}=+22.4^{\circ}, \varphi_{\text {long }}=+18^{\circ}$.

the temperature profile inward, making the conversion region cooler (this will be discussed in a future paper). If one expected a decrease in the emission intensity for low frequencies this could also arise from nonmonotonic density profiles in the scrape off layer (SOL) - which would block the emission (in SOL, unfortunately, there are no Thomson scattering data). However, for frequencies $f>16 \mathrm{GHz}$, one would not expect a strong decrease in the radiative temperature, which is observed in experiment. ${ }^{1}$ From Fig. 3, we see that there are no obstacles stopping the EBW at $16.5 \mathrm{GHz}$ from reaching the first harmonic at the plasma center.

The corresponding time development of EBWE at frequencies of 28 and $29 \mathrm{GHz}$ is shown in Fig. 6 as well as the Thomson scattering data. There is a substantial difference in the simulation signals - with significantly lower radiation temperature at $29 \mathrm{GHz}$ over than at $28 \mathrm{GHz}$. This difference between EBWE at these two frequencies can be understood by noting from Fig. 3 that the $29 \mathrm{GHz}$ signal is only slightly below the third harmonic. For incident rays from the edge, this would correspond to absorption at the Doppler shifted broadened resonance located near the plasma edge. This is confirmed by the details in the central ray propagation at $29 \mathrm{GHz}$, Fig. 7. The ray undergoes damping as soon as it enters the plasma [since $\left(\omega-3 \omega_{\mathrm{ce}}\right) / k_{\|} v_{T} \leqslant 3$ ] and does not penetrate past $8 \mathrm{~cm}$ from the edge, where the temperature is cold. On the other hand, the central ray at $28 \mathrm{GHz}$ can readily reach the second harmonic near the plasma center where the temperature is high. The simulation shows that the results are quite sensitive to variations in the magnetic field: e.g., if the experimental magnetic field is somewhat greater at the plasma boundary than that given by EFIT02, ${ }^{6}$ (e.g., by $2 \%$ ), then the $29 \mathrm{GHz}$ ray will now penetrate deep into the plasma and thus lead to strong absorption/emission.

The simulation frequency spectrum of EBWE for the frequency range $22 \mathrm{GHz} \leqslant f \leqslant 33 \mathrm{GHz}$ (appropriate for the high frequency antenna recently installed on NSTX) is shown in Fig. 8. The gap in emission at $31 \mathrm{GHz}$ is confirmed by Fig. 3 where one sees the crossing of the third harmonic 


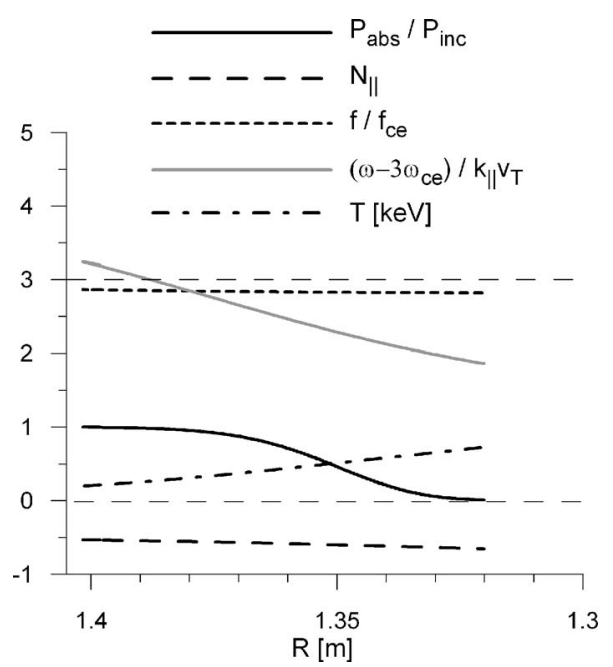

FIG. 7. The radial dependence of various parameters along the central ray as it propagates from the plasma edge $(R=1.4 \mathrm{~m})$ for shot 12078 at $29 \mathrm{GHz}$; angles are given in Fig. 6 and $t=0.365 \mathrm{~s}$. Power absorption/emission is very localized near the plasma edge $(R=1.33 \mathrm{~m})$ where the temperature is low since $\left(\omega-3 \omega_{\text {ce }}\right) / k_{\|} v_{T} \leqslant 3$.

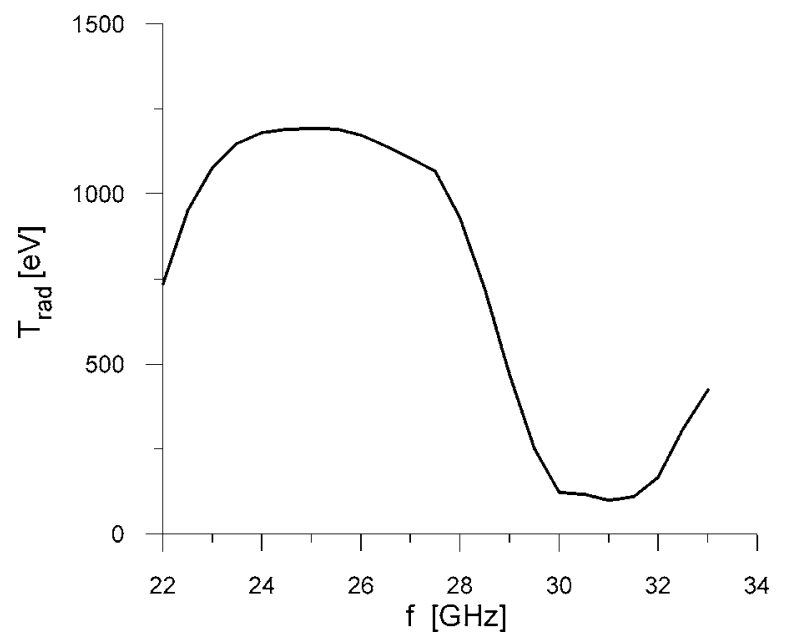

FIG. 8. The simulation frequency spectrum of EBWE for the NSTX high frequency antenna range. Angles are given in Fig. 6 at $t=0.382 \mathrm{~s}$. with the upper hybrid resonance. For the lower frequencies in the $23-28 \mathrm{GHz}$ range, the simulated emission from the second harmonic near the plasma center is high and quite flat and corresponds to the plasma properties shown in Fig. 3.

\section{SUMMARY}

There is a qualitative agreement between experimental and simulation results on antenna aiming for $L$-mode plasmas. This has been tested for several shots, including some $\mathrm{H}$-mode plasmas. During flattop current discharges it seems that the optimal angles are quite rugged and are independent of time. There is a good agreement in the time development of the emission for the low frequency antenna between simulation and experiment. The simulated frequency dependence of the emission shows some deviations from experiment for the low frequency antenna, but this should be corrected by inclusion of collisional damping corresponding to high $Z_{\text {eff }}$ in helium discharges.

For the high frequency antenna, the final experimental results are being recalibrated and so are not yet available for comparison with simulated ECE. This comparison will be postponed to a future paper.

\section{ACKNOWLEDGMENTS}

The authors thank A. S. Sabbagh for discussions concerning the EFIT in NSTX. This work was supported by the U.S. Department of Energy and by AS-CR Project No. AV0Z-20430508.

${ }^{1}$ S. J. Diem et al., Rev. Sci. Instrum. 77 (2006).

${ }^{2}$ L. L. Lao, H. Stjohn, R. D. Stambaugh, A. G. Kellman, and W. Pfeiffer, Nucl. Fusion 25, 1611 (1985).

${ }^{3}$ J. Urban and J. Preinhaelter, Czech. J. Phys. 54, Suppl. C, C109 (2004).

${ }^{4}$ P. Pavlo and L. Krlin, Nucl. Fusion 31, 711 (1991).

${ }^{5}$ M. Bornatici, R. Cano, O. Debarbieri, and F. Engelmann, Nucl. Fusion 23, 1153 (1983).

${ }^{6}$ S. A. Sabbagh et al., Nucl. Fusion 41, 1601 (2001); Nucl. Fusion 46, 635 (2006).

${ }^{7}$ T. H. Stix, Waves in Plasmas (AIP, New York, 1992).

${ }^{8}$ PHYTON, http://www.phyton.org 